\title{
ON WEIGHTED INEQUALITIES FOR PARAMETRIC MARCINKIEWICZ INTEGRALS
}

\author{
H. M. AL-QASSEM
}

Received 25 February 2005; Revised 30 May 2005; Accepted 3 July 2005

We establish a weighted $L^{p}$ boundedness of a parametric Marcinkiewicz integral operator $M_{\Omega, h}^{\rho}$ if $\Omega$ is allowed to be in the block space $B_{q}^{(0,-1 / 2)}\left(S^{n-1}\right)$ for some $q>1$ and $h$ satisfies a mild integrability condition. We apply this conclusion to obtain the weighted $L^{p}$ boundedness for a class of the parametric Marcinkiewicz integral operators $\mathcal{M}_{\Omega, h, \lambda}^{*, \rho}$ and $M_{\Omega, h, S}^{\rho}$ related to the Littlewood-Paley $g_{\lambda}^{*}$-function and the area integral $S$, respectively. It is known that the condition $\Omega \in B_{q}^{(0,-1 / 2)}\left(\mathbf{S}^{n-1}\right)$ is optimal for the $L^{2}$ boundedness of $M_{\Omega, 1}^{1}$.

Copyright (c) 2006 H. M. Al-Qassem. This is an open access article distributed under the Creative Commons Attribution License, which permits unrestricted use, distribution, and reproduction in any medium, provided the original work is properly cited.

\section{Introduction}

Suppose that $\mathbf{S}^{n-1}$ is the unit sphere of $\mathbf{R}^{n}(n \geq 2)$ equipped with the normalized Lebesgue measure $d \sigma=d \sigma(\cdot)$. Let $\Omega$ be a function defined on $\mathbf{S}^{n-1}$ with $\Omega \in L^{1}\left(\mathbf{S}^{n-1}\right)$ and satisfies the vanishing condition

$$
\int_{S^{n-1}} \Omega(x) d \sigma(x)=0 .
$$

For $\gamma>1$, let $\Delta_{\gamma}\left(\mathbf{R}^{+}\right)$denote the set of all measurable functions $h$ on $\mathbf{R}^{+}$such that

$$
\sup _{R>0} \frac{1}{R} \int_{0}^{R}|h(t)|^{\gamma} d t<\infty .
$$

It is easy to see that the following inclusions hold and are proper:

$$
L^{\infty}\left(\mathbf{R}^{+}\right) \subset \Delta_{\beta}\left(\mathbf{R}^{+}\right) \subset \Delta_{\alpha}\left(\mathbf{R}^{+}\right) \quad \text { for } \alpha<\beta .
$$

Throughout this paper, we let $x^{\prime}$ denote $x /|x|$ for $x \in \mathbf{R}^{n} \backslash\{0\}$ and $p^{\prime}$ denote the conjugate index of $p$; that is, $1 / p+1 / p^{\prime}=1$. 
Suppose that $\Gamma(t)$ is a strictly monotonic $C^{1}$ function on $\mathbf{R}_{+}$and $h: \mathbf{R}_{+} \rightarrow \mathbf{C}$ is a measurable function. Define the parametric Marcinkiewicz integral operator $\mu_{\Omega, \Gamma, h}^{\rho}$ by

$$
\mathcal{M}_{\Omega, \Gamma, h}^{\rho} f(x)=\left(\int_{0}^{\infty}\left|F_{\Omega, \Gamma, h}^{\rho} f(t, x)\right|^{2} \frac{d t}{t}\right)^{1 / 2},
$$

where

$$
F_{\Omega, \Gamma, h}^{\rho} f(t, x)=\frac{1}{t^{\rho}} \int_{|u| \leq t} f\left(x-\Gamma(|u|) u^{\prime}\right) \frac{\Omega\left(u^{\prime}\right)}{|u|^{n-\rho}} h(|u|) d u
$$

$\rho=\sigma+i \tau(\sigma, \tau \in \mathbf{R}$ with $\sigma>0), f \in \mathscr{S}\left(\mathbf{R}^{n}\right)$, the space of Schwartz functions.

For the sake of simplicity, we denote $\mathcal{M}_{\Omega, \Gamma, h}^{\rho}=\mathcal{M}_{\Omega, h}^{\rho}$ if $\Gamma(t) \equiv t$.

It is well-known that $M_{\Omega, 1}^{1}$ is the classical Marcinkiewicz integral operator of higher dimension, corresponding to the Littlewood-Paley $g$-function, introduced by Stein in [17]. Stein showed that $M_{\Omega, 1}^{1}$ is bounded on $L^{p}\left(\mathbf{R}^{n}\right)$ for $p \in(1,2]$ if $\Omega \in \operatorname{Lip}_{\alpha}\left(\mathbf{S}^{n-1}\right)(0<\alpha \leq 1)$. Subsequently, Benedek et al. proved that $M_{\Omega, 1}^{1}$ is bounded on $L^{p}\left(\mathbf{R}^{n}\right)$ for $p \in(1, \infty)$ if $\Omega \in C^{1}\left(\mathbf{S}^{n-1}\right)$ (see [3]). Later on, the case of rough kernels ( $\Omega$ satisfies only size and cancelation conditions but no regularity is assumed) became the interest of many authors. For a sample of past studies, see $([1,2,4,5])$. In [2], Al-Qassem and Al-Salman showed that $M_{\Omega, 1}^{1}$ is bounded on $L^{p}\left(\mathbf{R}^{n}\right)$ for $p \in(1, \infty)$ if $\Omega$ belongs to the block space $B_{q}^{(0,-1 / 2)}\left(\mathbf{S}^{n-1}\right)$ and that the condition $\Omega \in B_{q}^{(0,-1 / 2)}\left(\mathbf{S}^{n-1}\right)$ is optimal in the sense that there exists an $\Omega$ which lies in $B_{q}^{(0, v)}\left(\mathbf{S}^{n-1}\right)$ for all $-1<v<-1 / 2$ such that $M_{\Omega, 1}^{1}$ is not bounded on $L^{2}\left(\mathbf{R}^{n}\right)$. In Hörmander [10] defined the parametric Marcinkiewicz operator $\mathcal{M}_{\Omega, 1}^{\rho}$ for $\rho>0$ and proved that $\mathcal{M}_{\Omega, 1}^{1}$ is bounded on $L^{p}\left(\mathbf{R}^{n}\right)$ for $p \in(1, \infty)$ if $\Omega \in \operatorname{Lip}_{\alpha}\left(\mathbf{S}^{n-1}\right)(0<\alpha \leq 1)$. Sakamoto and Yabuta [15] studied the $L^{p}$-boundedness of the more general parametric Marcinkiewicz integral operator $\mathcal{M}_{\Omega, 1}^{\rho}$ if $\rho$ is complex and proved that $M_{\Omega, 1}^{\rho}$ is bounded on $L^{p}\left(\mathbf{R}^{n}\right)$ for $p \in(1, \infty)$ if $\operatorname{Re}(\rho)=\sigma>0$ and $\Omega \in \operatorname{Lip}_{\alpha}\left(\mathbf{S}^{n-1}\right)(0<\alpha \leq 1)$. Recently, in [1] the author of this paper gave that the more general operator $M_{\Omega, \Gamma, h}^{\rho}$ is bounded on $L^{p}\left(\mathbf{R}^{n}\right)$ for $p$ satisfying $|1 / p-1 / 2| \leq \min \left\{1 / 2,1 / \gamma^{\prime}\right\}$ if $\operatorname{Re}(\rho)=\sigma>0$, $\Gamma$ satisfies a convexity condition, $\Omega \in B_{q}^{(0,-1 / 2)}\left(\mathbf{S}^{n-1}\right)$ and $h \in \Delta_{\gamma}\left(\mathbf{R}^{+}\right)$for some $q, \gamma>1$. This is an essential improvement and extension of the results mentioned above.

On the other hand, the weighted $L^{p}$ boundedness of $M_{\Omega, h}^{1}$ has also attracted the attention of many authors in the recent years. Indeed, Torchinsky and Wang in [19] proved that if $\Omega \in \operatorname{Lip}_{\alpha}\left(\mathbf{S}^{n-1}\right),(0<\alpha \leq 1)$, then $\mathcal{M}_{\Omega, 1}^{1}$ is bounded on $L^{p}(\omega)$ for $p \in(1, \infty)$ and $\omega \in A_{p}$ (The Muckenhoupt's weight class, see [9] for the definition). In Sato in [16] improved the weighted $L^{p}$ boundedness of Torchinsky-Wang by proving that $M_{\Omega, h}^{1}$ is bounded on $L^{p}(\omega)$ for $p \in(1, \infty)$ provided that $h \in L^{\infty}\left(\mathbf{R}_{+}\right), \Omega \in L^{\infty}\left(\mathbf{S}^{n-1}\right)$ and $\omega \in A_{p}\left(\mathbf{R}^{n}\right)$. Subsequently, in Ding et al. in [5] were able to show that $M_{\Omega, h}^{1}$ is bounded on $L^{p}(\omega)$ for $p \in(1, \infty)$ provided that $h \in L^{\infty}\left(\mathbf{R}_{+}\right), \Omega \in L^{q}\left(\mathbf{S}^{n-1}\right), q>1$ and $\omega^{q^{\prime}} \in A_{p}\left(\mathbf{R}^{n}\right)$. In a recent paper, Lee and Lin in [13] showed that $M_{\Omega, h}^{1}$ is bounded on $L^{p}(\omega)$ for $p \in(1, \infty)$ if $h \in L^{\infty}\left(\mathbf{R}_{+}\right)$, $\Omega \in H^{1}\left(\mathbf{S}^{n-1}\right)$ and $\omega \in \widetilde{A}_{p}^{I}\left(\mathbf{R}^{n}\right)$, where $H^{1}\left(\mathbf{S}^{n-1}\right)$ is the Hardy space on the unit sphere and $\widetilde{A}_{p}^{I}\left(\mathbf{R}^{n}\right)$ is a special class of radial weights introduced by Duoandikoetxea [6] whose definition will recalled in Section 2. 
In this paper, we will investigate the weighted $L^{p}(\omega)$ boundedness of the parametric Marcinkiewicz operator $\mathcal{M}_{\Omega, \Gamma, h}^{\rho}$ for $\omega \in \widetilde{A}_{p}^{I}\left(\mathbf{R}^{n}\right)$ and under the natural condition $\Omega \in$ $B_{q}^{(0,-1 / 2)}\left(\mathbf{S}^{n-1}\right)$. To state our results, we will need the following definitions from [8].

Definition 1.1. We say that a function $\Gamma$ satisfies "hypothesis I" if

(a) $\Gamma$ is a nonnegative $C^{1}$ function on $(0, \infty)$,

(b) $\Gamma$ is strictly increasing, $\Gamma(2 t) \geq \eta \Gamma(t)$ for some fixed $\eta>1$ and $\Gamma(2 t) \leq c \Gamma(t)$ for some constant $c \geq \eta>1$.

(c) $\Gamma^{\prime}(t) \geq \alpha \Gamma(t) / t$ on $(0, \infty)$ for some fixed $\alpha \in\left(0, \log _{2} c\right]$ and $\Gamma^{\prime}(t)$ is monotone on $(0, \infty)$.

Definition 1.2. We say that $\Gamma$ satisfies "hypothesis $D$ " if

$\left(\mathrm{a}^{\prime}\right) \Gamma$ is a nonnegative $C^{1}$ function on $(0, \infty)$,

$\left(\mathrm{b}^{\prime}\right) \Gamma$ is strictly decreasing, $\Gamma(t) \geq \eta \Gamma(2 t)$ for some fixed $\eta>1$ and $\Gamma(t) \leq c \Gamma(2 t)$ for some constant $c \geq \eta>1$.

(c $\left.c^{\prime}\right)\left|\Gamma^{\prime}(t)\right| \geq \alpha \Gamma(t) / t$ on $(0, \infty)$ for some fixed $\alpha \in\left(0, \log _{2} c\right]$ and $\Gamma^{\prime}(t)$ is monotone on $(0, \infty)$.

Model functions for the $\Gamma$ satisfy hypothesis I are $\Gamma(t)=t^{d}$ with $d>0$, and their linear combinations with positive coefficients. Model functions for the $\Gamma$ satisfy hypothesis $\mathbf{D}$ are $\Gamma(t)=t^{r}$ with $r<0$, and their linear combinations with positive coefficients.

Theorem 1.3. Let $h \in \Delta_{\gamma}\left(\mathbf{R}_{+}\right)$for some $\gamma>1$. Assume that $\Gamma$ satisfies either hypothesis $\mathbf{I}$ or hypothesis $\mathbf{D}$ and $\Omega \in B_{q}^{(0,-1 / 2)}\left(\mathbf{S}^{n-1}\right)$ for some $q>1$. Then

$$
\left\|M_{\Omega, \Psi, h}^{\rho}(f)\right\|_{L^{p}\left(\mathbf{R}^{n}\right)} \leq C_{p}\|\Omega\|_{B_{q}^{(0,-1 / 2)}\left(\mathbf{S}^{n-1}\right)}\|f\|_{L^{p}\left(\mathbf{R}^{n}\right)}
$$

is bounded on $L^{p}\left(\mathbf{R}^{n}\right)$ for $|1 / p-1 / 2|<\min \left\{1 / \gamma^{\prime}, 1 / 2\right\}$.

Theorem 1.4. Let $h \in \Delta_{\gamma}\left(\mathbf{R}_{+}\right)$for some $\gamma \geq 2,1<p<\infty$. Assume that $\Gamma$ satisfies either hypothesis $\mathbf{I}$ or hypothesis $\mathbf{D}$ and $\Omega \in B_{q}^{(0,-1 / 2)}\left(\mathbf{S}^{n-1}\right)$ for some $q>1$. Then there exists $C_{p}>0$ such that the following inequality holds:

$$
\left\|M_{\Omega, \Gamma, h}^{\rho}(f)\right\|_{L^{p}(\omega)} \leq C_{p}\|\Omega\|_{B_{q}^{(0,-1 / 2)}\left(\mathbf{S}^{n-1}\right)}\|f\|_{L^{p}(\omega)}
$$

for $\gamma^{\prime}<p<\infty$ and $\omega \in \tilde{A}_{p / \gamma^{\prime}}^{I}\left(\mathbf{R}_{+}\right)$.

Remark 1.5. (a) In order to make a comparison among the above mentioned results, we remark that on $S^{n-1}$, for any $q>1,0<\alpha \leq 1$ and $-1<v$, the following inclusions hold and are proper:

$$
\begin{gathered}
C^{1}\left(\mathbf{S}^{n-1}\right) \subset \operatorname{Lip}_{\alpha}\left(\mathbf{S}^{n-1}\right) \subset L^{q}\left(\mathbf{S}^{n-1}\right) \subset L\left(\log ^{+} L\right)\left(\mathbf{S}^{n-1}\right) \subset H^{1}\left(\mathbf{S}^{n-1}\right), \\
\bigcup_{r>1} L^{r}\left(\mathbf{S}^{n-1}\right) \subset B_{q}^{(0, v)}\left(\mathbf{S}^{n-1}\right) .
\end{gathered}
$$

With regard to the relationship between $B_{q}^{(0, v)}\left(\mathbf{S}^{n-1}\right)$ and $H^{1}\left(\mathbf{S}^{n-1}\right)($ for $v>-1)$ remains open.

(b) We point out that the result in Theorem 1.3 extends the result of Al-Qassem and Al-Salman [2] who obtained Theorem 1.3 in the special case $h \equiv 1$ and $\Gamma(t) \equiv t$ and 
also improves substantially the result of Sakamoto and Yabuta [15]. We remark also that Theorem 1.4 represents an improvement and extension of [5, Theorem 1] in the case $\omega \in \tilde{A}_{p}^{I}\left(\mathbf{R}_{+}\right)$.

(c) The method employed in this paper is based in part on ideas from $[1,2,7,8,16]$, among others.

The paper is organized as follows. In Section 2 we give some definitions and we establish the main estimates needed in the proofs of our main results. The proofs of Theorems 1.3 and 1.4 will be given in Section 3. Additional results can be found in Section 4 .

Throughout the rest of the paper the letter $C$ will denote a positive constant whose value may change at each occurrence.

\section{Definitions and lemmas}

Let us begin by recalling the definition of some special classes of weights and some of their important properties.

Definition 2.1. Let $\omega(t) \geq 0$ and $\omega \in L_{\mathrm{loc}}^{1}\left(\mathbf{R}_{+}\right)$. For $1<p<\infty$, we say that $\omega \in A_{p}\left(\mathbf{R}_{+}\right)$if there is a positive constant $C$ such that for any interval $I \subset \mathbf{R}_{+}$,

$$
\left(|I|^{-1} \int_{I} \omega(t) d t\right)\left(|I|^{-1} \int_{I} \omega(t)^{-1 /(p-1)} d t\right)^{p-1} \leq C<\infty .
$$

$A_{1}\left(\mathbf{R}_{+}\right)$is the class of weights $\omega$ for which $M$ satisfies a weak-type estimate in $L^{1}(\omega)$, where $M(f)$ is the Hardy-Littlewood maximal function of $f$.

It is well-known that the class $A_{1}\left(\mathbf{R}_{+}\right)$is also characterized by all weights $\omega$ for which $M \omega(t) \leq C \omega(t)$ for a.e. $t \in \mathbf{R}_{+}$and for some positive constant $C$.

Definition 2.2. Let $1 \leq p<\infty$. We say that $\omega \in \widetilde{A}_{p}\left(\mathbf{R}_{+}\right)$if

$$
\omega(x)=v_{1}(|x|) \nu_{2}(|x|)^{1-p},
$$

where either $\nu_{i} \in A_{1}\left(\mathbf{R}_{+}\right)$is decreasing or $v_{i}^{2} \in A_{1}\left(\mathbf{R}_{+}\right), i=1,2$.

Let $A_{p}^{I}\left(\mathbf{R}^{n}\right)$ be the weight class defined by exchanging the cubes in the definitions of $A_{p}$ for all $n$-dimensional intervals with sides parallel to coordinate axes (see [12]). Let $\widetilde{A}_{p}^{I}=\widetilde{A}_{p} \cap A_{p}^{I}$. If $\omega \in \widetilde{A}_{p}$, it follows from [6] that the classical Hardy-Littlewood maximal function $M f$ is bounded on $L^{p}\left(\mathbf{R}^{n}, \omega(|x|) d x\right)$. Therefore, if $\omega(t) \in \widetilde{A}_{p}\left(\mathbf{R}_{+}\right)$, then $\omega(|x|) \in$ $A_{p}\left(\mathbf{R}^{n}\right)$.

By following the same argument as in the proof of the elementary properties of $A_{p}$ weight class (see, e.g., [9]) we get the following lemma.

Lemma 2.3. If $1 \leq p<\infty$, then the weight class $\tilde{A}_{p}^{I}\left(\mathbf{R}_{+}\right)$has the following properties:

(i) $\widetilde{A}_{p_{1}}^{I} \subset \widetilde{A}_{p_{1}}^{I}$, if $1 \leq p_{1}<p_{2}<\infty$;

(ii) For any $\omega \in \widetilde{A}_{p}^{I}$, there exists an $\varepsilon>0$ such that $\omega^{1+\varepsilon} \in \tilde{A}_{p}^{I}$;

(iii) For any $\omega \in \widetilde{A}_{p}^{I}$ and $p>1$, there exists an $\varepsilon>0$ such that $p-\varepsilon>1$ and $\omega \in \widetilde{A}_{p-\varepsilon}^{I}$.

The block spaces originated in the work of Taibleson and Weiss on the convergence of the Fourier series in connection with the developments of the real Hardy spaces. Below 
we will recall the definition of block spaces on $\mathbf{S}^{n-1}$. For further background information about the theory of spaces generated by blocks and its applications to harmonic analysis, see the book [14].

Definition 2.4. A $q$-block on $S^{n-1}$ is an $L^{q}(1<q \leq \infty)$ function $b(x)$ that satisfies

$$
\begin{aligned}
& \text { (i) } \operatorname{supp}(b) \subset I \\
& \text { (ii) }\|b\|_{L^{q}} \leq|I|^{-1 / q^{\prime}},
\end{aligned}
$$

where $|I|=\sigma(I)$, and $I=B\left(x_{0}^{\prime}, \theta_{0}\right)=\left\{x^{\prime} \in \mathbf{S}^{n-1}:\left|x^{\prime}-x_{0}^{\prime}\right|<\theta_{0}\right\}$ is a cap on $\mathbf{S}^{n-1}$ for some $x_{0}^{\prime} \in \mathbf{S}^{n-1}$ and $\theta_{0} \in(0,1]$.

Jiang and Lu introduced (see [14]) the class of block spaces $B_{q}^{(0, v)}\left(\mathbf{S}^{n-1}\right)$ (for $v>-1$ ) with respect to the study of homogeneous singular integral operators.

Definition 2.5. The block space $B_{q}^{(0, v)}\left(\mathbf{S}^{n-1}\right)$ is defined by

$$
B_{q}^{(0, v)}\left(\mathbf{S}^{n-1}\right)=\left\{\Omega \in L^{1}\left(\mathbf{S}^{n-1}\right): \Omega=\sum_{\mu=1}^{\infty} \eta_{\mu} b_{\mu}, M_{q}^{(0, v)}\left(\left\{\eta_{\mu}\right\}\right)<\infty\right\},
$$

where each $\eta_{\mu}$ is a complex number; each $b_{\mu}$ is a $q$-block supported on a cap $I_{\mu}$ on $\mathbf{S}^{n-1}$, $v>-1$ and

$$
M_{q}^{(0, v)}\left(\left\{\eta_{\mu}\right\}\right)=\sum_{\mu=1}^{\infty}\left|\eta_{\mu}\right|\left\{1+\log ^{(v+1)}\left(\left|I_{\mu}\right|^{-1}\right)\right\}
$$

Let $\|\Omega\|_{B_{q}^{(0, v)}\left(\mathbf{S}^{n-1}\right)}=\inf \left\{M_{q}^{(0, v)}\left(\left\{\eta_{\mu}\right\}\right): \Omega=\sum_{\mu=1}^{\infty} \eta_{\mu} b_{\mu}\right.$ and each $b_{\mu}$ is a $q$-block function supported on a cap $I_{\mu}$ on $\left.\mathbf{S}^{n-1}\right\}$. Then $\|\cdot\|_{B_{q}^{(0, v)}\left(\mathbf{S}^{n-1}\right)}$ is a norm on the space $B_{q}^{(0, v)}\left(\mathbf{S}^{n-1}\right)$ and $\left(B_{q}^{(0, v)}\left(\mathbf{S}^{n-1}\right),\|\cdot\|_{B_{q}^{(0, v)}\left(\mathbf{S}^{n-1}\right)}\right)$ is a Banach space.

In their investigations of block spaces, Keitoku and Sato in [11] showed that these spaces enjoy the following properties:

$$
\begin{gathered}
B_{q}^{\left(0, v_{2}\right)}\left(\mathbf{S}^{n-1}\right) \subset B_{q}^{\left(0, v_{1}\right)}\left(\mathbf{S}^{n-1}\right) \quad \text { if } v_{2}>v_{1}>-1 ; \\
B_{q_{2}}^{(0, v)}\left(\mathbf{S}^{n-1}\right) \subset B_{q_{1}}^{(0, v)}\left(\mathbf{S}^{n-1}\right) \quad \text { if } 1<q_{1}<q_{2}, \text { for any } v>-1 ; \\
\bigcup_{q>1} B_{q}^{(0, v)}\left(\mathbf{S}^{n-1}\right) \nsubseteq \bigcup_{q>1} L^{q}\left(\mathbf{S}^{n-1}\right) \quad \text { for any } v>-1 .
\end{gathered}
$$

Definition 2.6. For a suitable $C^{1}$ function $\Gamma$ on $\mathbf{R}_{+}$, a measurable function $h: \mathbf{R}_{+} \rightarrow \mathbf{C}$ and a suitable function $\tilde{b}_{\mu}$ on $\mathbf{S}^{n-1}$ we define the family of measures $\left\{\sigma_{\tilde{b}_{\mu}, t}: t \in \mathbf{R}_{+}\right\}$and the maximal operator $\sigma_{\widetilde{b}_{\mu}}^{*}$ on $\mathbf{R}^{n}$ by

$$
\begin{aligned}
\int_{\mathbf{R}^{n}} f d \sigma_{\widetilde{b}_{\mu}, t}= & \frac{1}{t^{\rho}} \int_{(1 / 2) t<|y| \leq t} f\left(\Gamma(|y|) y^{\prime}\right) h(|y|) \frac{\widetilde{b}_{\mu}\left(y^{\prime}\right)}{|y|^{n-\rho}} d y, \\
& \sigma_{\widetilde{b}_{\mu}}^{*} f(x)=\sup _{t \in \mathbf{R}_{+}}|| \sigma_{\widetilde{b}_{\mu}, t}|* f(x)|
\end{aligned}
$$


where $\left|\sigma_{\widetilde{b}_{\mu}, t}\right|$ is defined in the same way as $\sigma_{\widetilde{b}_{\mu}, t}$, but with $\tilde{b}_{\mu}$ replaced by $\left|\tilde{b}_{\mu}\right|$ and $h$ replaced by $|h|$.

For $k \in \mathbf{Z}, \mu \in \mathbf{N} \cup\{0\}$, and a cap $I_{\mu}$ on $\mathbf{S}^{n-1}$ with $\left|I_{\mu}\right|<e^{-2}$, we let $\theta_{\mu}=\left[\log \left|I_{\mu}\right|^{-1}\right]$ and $\omega_{\mu}=2^{\theta_{\mu}}$, where $[\cdot]$ denotes the greatest integer function. Now set $a_{k, \mu}=\Gamma\left(\omega_{\mu}^{k}\right)$ if $\Gamma$ satisfies hypothesis I and $a_{k, \mu}=\left(\Gamma\left(\omega_{\mu}^{k}\right)\right)^{-1}$ if $\Gamma$ satisfies hypothesis $\mathbf{D}$. Then by the conditions of $\Gamma$, it is easy to see that $\left\{a_{k, \mu}\right\}$ is a lacunary sequence of positive numbers with $\inf _{k \in \mathbf{Z}}\left(a_{k+1, \mu} / a_{k, \mu}\right) \geq \eta^{\theta_{\mu}}>1$.

Lemma 2.7. Let $\mu \in \mathbf{N} \cup\{0\}$ and $h \in \Delta_{\gamma}\left(\mathbf{R}_{+}\right)$for some $\gamma$ with $1<\gamma \leq 2$. Let $\tilde{b}_{\mu}$ be a function on $\mathbf{S}^{n-1}$ satisfying (i) $\int_{\mathbf{S}^{n-1}} \tilde{b}_{\mu}(y) d \sigma(y)=0$; (ii) $\left\|\tilde{b}_{\mu}\right\|_{q} \leq\left|I_{\mu}\right|^{-1 / q^{\prime}}$ for some $q>1$ and for some cap $I_{\mu}$ on $\mathbf{S}^{n-1}$ with $\left|I_{\mu}\right|<e^{-2}$; and (iii) $\left\|\tilde{b}_{\mu}\right\|_{1} \leq 1$. Then there exist constants $C$ and $0<v<1 / q^{\prime}$ such that if $\Gamma$ satisfies hypothesis $\mathbf{I}$,

$$
\begin{gathered}
\left\|\sigma_{\widetilde{b}_{\mu}, t}\right\| \leq C ; \\
\int_{\omega_{\mu}^{k}}^{\omega_{\mu}^{k+1}}\left|\hat{\sigma}_{\widetilde{b}_{\mu}, t}(\xi)\right|^{2} \frac{d t}{t} \leq C \theta_{\mu}\left(a_{k, \mu}\right)^{-2 v / \gamma^{\prime} \theta_{\mu}}|\xi|^{-2 v / \gamma^{\prime} \theta_{\mu}} ; \\
\int_{\omega_{\mu}^{k}}^{\omega_{\mu}^{k+1}}\left|\hat{\sigma}_{\widetilde{b}_{\mu}, t}(\xi)\right|^{2} \frac{d t}{t} \leq C \theta_{\mu}\left(a_{k, \mu}\right)^{2 v / \gamma^{\prime} \theta_{\mu}}|\xi|^{2 v / \gamma^{\prime} \theta_{\mu}},
\end{gathered}
$$

and if $\Gamma$ satisfies hypothesis $\mathbf{D}$,

$$
\begin{gathered}
\left\|\sigma_{\widetilde{b}_{\mu}, t}\right\| \leq C ; \\
\int_{\omega_{\mu}^{k}}^{\omega_{\mu}^{k+1}}\left|\hat{\sigma}_{\widetilde{b}_{\mu}, t}(\xi)\right|^{2} \frac{d t}{t} \leq C \theta_{\mu}\left(a_{k, \mu}\right)^{-2 v / \gamma^{\prime} \theta_{\mu}}|\xi|^{2 v / \gamma^{\prime} \theta_{\mu}} ; \\
\int_{\omega_{\mu}^{k}}^{\omega_{\mu}^{k+1}}\left|\hat{\sigma}_{\widetilde{b}_{\mu}, t}(\xi)\right|^{2} \frac{d t}{t} \leq C \theta_{\mu}\left(a_{k, \mu}\right)^{2 v / \gamma^{\prime} \theta_{\mu}}|\xi|^{-2 v / \gamma^{\prime} \theta_{\mu}},
\end{gathered}
$$

where $\left\|\sigma_{\widetilde{b}_{\mu}, t}\right\|$ stands for the total variation of $\sigma_{\widetilde{b}_{\mu}, t}$. The constant $C$ is independent of $k, \mu, \xi$ and $\Gamma(\cdot)$.

Proof. We will only present the proof of the lemma if $\Gamma$ satisfies hypothesis $\mathbf{I}$, since the proof for the case that $\Gamma$ satisfies hypothesis $\mathbf{D}$ will be essentially the same. By (iii) and the definition of $\sigma_{\widetilde{b}_{\mu}, t}$, one can easily see that (2.8) holds with a constant $C$ independent of $t$ and $\mu$. Next we prove (2.9). By definition,

$$
\widehat{\sigma}_{\tilde{b}_{\mu}, t}(\xi)=\frac{1}{t^{\rho}} \int_{(1 / 2) t}^{t} \int_{S^{n-1}} e^{-i \Psi(s) \xi \cdot x} \tilde{b}_{\mu}(x) \frac{h(s)}{s^{1-\rho}} d \sigma(x) d s .
$$


By Hölder's inequality, a change of variable and since $\left|\int_{S^{n-1}} e^{-i \Psi(s) \xi \cdot x} \tilde{b}_{\mu}(x) d \sigma(x)\right| \leq 1$, we obtain

$$
\begin{aligned}
\left|\hat{\sigma}_{\tilde{b}_{\mu}, t}(\xi)\right| & \leq\left(\int_{(1 / 2) t}^{t}|h(s)|^{\gamma} \frac{d s}{s}\right)^{1 / \gamma}\left(\int_{(1 / 2) t}^{t}\left|\int_{S^{n-1}} e^{-i \Psi(s) \xi \cdot x} \tilde{b}_{\mu}(x) d \sigma(x)\right|^{\gamma^{\prime}} \frac{d s}{s}\right)^{1 / \gamma^{\prime}} \\
& \leq C\left(\int_{(1 / 2) t}^{t}\left|\int_{S^{n-1}} e^{-i \Psi(s) \xi \cdot x} \tilde{b}_{\mu}(x) d \sigma(x)\right|^{2} \frac{d s}{s}\right)^{1 / \gamma^{\prime}} \\
& =C\left(\int_{S^{n-1} \times S^{n-1}} \tilde{b}_{\mu}(x) \overline{\tilde{b}_{\mu}(y)} I_{\mu, t}(\xi, x, y) d \sigma(x) d \sigma(y)\right)^{1 / \gamma^{\prime}}
\end{aligned}
$$

where

$$
I_{\mu, t}(\xi, x, y)=\int_{1 / 2}^{1} e^{-i \Gamma(t s) \xi \cdot(x-y)} \frac{d s}{s} .
$$

Write $I_{\mu, t}(\xi, x, y)$ as

$$
I_{\mu, t}(\xi, x, y)=\int_{1 / 2}^{1} Y_{t}^{\prime}(s) \frac{d s}{s}
$$

where

$$
Y_{t}(s)=\int_{1 / 2}^{s} e^{-i \Gamma(t w) \xi \cdot(x-y)} d w, \quad 1 / 2 \leq s \leq 1
$$

Now, using the assumptions on $\Gamma$, we obtain

$$
\frac{d}{d w}(\Gamma(t w))=t \Gamma^{\prime}(t w) \geq \alpha \frac{\Gamma(t w)}{w} \geq \alpha \frac{\Gamma(t / 2)}{s} \geq \frac{\alpha}{c} \frac{\Gamma(t)}{s} \quad \text { for } 1 / 2 \leq w \leq s \leq 1 .
$$

Thus by van der Corput's lemma, $\left|Y_{t}(s)\right| \leq(c / \alpha)|\Gamma(t) \xi / s|^{-1}\left|\xi^{\prime} \cdot(x-y)\right|^{-1}$. By integration by parts, we get

$$
\left|I_{\mu, t}(\xi, x, y)\right| \leq C|\Gamma(t) \xi|^{-1}\left|\xi^{\prime} \cdot(x-y)\right|^{-1}
$$

which when combined with the trivial estimate $\left|I_{\mu, t}(\xi, x, y)\right| \leq \log 2$ and choosing $\tau$ such that $0<\tau<1 / q^{\prime}$ yields

$$
\left|I_{\mu, t}(\xi, x, y)\right| \leq|\Gamma(t) \xi|^{-\tau}\left|\xi^{\prime} \cdot(x-y)\right|^{-\tau}
$$

By Hölder's inequality and (ii) we get

$$
\begin{aligned}
\left|\hat{\sigma}_{\widetilde{b}_{\mu}, t}(\xi)\right| \leq & C|\Gamma(t) \xi|^{-\tau / \gamma^{\prime}}|| \tilde{b}_{\mu} \|_{q}^{2 / \gamma^{\prime}} \\
& \times\left(\int_{S^{n-1} \times \mathbf{S}^{n-1}}\left|\xi^{\prime} \cdot(x-y)\right|^{-\tau q^{\prime}} d \sigma(x) d \sigma(y)\right)^{1 /\left(q^{\prime} \gamma^{\prime}\right)} \\
\leq & C|\Gamma(t) \xi|^{-\tau / \gamma^{\prime}}\left|I_{\mu}\right|^{-2 /\left(q^{\prime} \gamma^{\prime}\right)} .
\end{aligned}
$$


8 Weighted marcinkiewicz integrals

Therefore,

$$
\begin{aligned}
\int_{\omega_{\mu}^{k}}^{\omega_{\mu}^{k+1}}\left|\widehat{\sigma}_{\widetilde{b}_{\mu}, t}(\xi)\right|^{2} \frac{d t}{t} & \leq C \min \left\{\log \left(\left|I_{\mu}\right|^{-1}\right), \log \left(\left|I_{\mu}\right|^{-1}\right)\left|\Gamma\left(\omega_{\mu}^{k}\right) \xi\right|^{-2 \tau / \gamma^{\prime}}\left|I_{\mu}\right|^{-2 /\left(q^{\prime} \gamma^{\prime}\right)}\right\} \\
& \leq C \log \left(\left|I_{\mu}\right|^{-1}\right)\left|\Gamma\left(\omega_{\mu}^{k}\right) \xi\right|^{-2 \tau / \gamma^{\prime} \log \left(\left|I_{\mu}\right|^{-1}\right)},
\end{aligned}
$$

which proves (2.9). To prove (2.10), we use the cancellation condition of $\tilde{b}_{\mu}$ to get

$$
\left|\hat{\sigma}_{\tilde{b}_{\mu}, t}(\xi)\right| \leq \int_{S^{n-1}} \int_{t / 2}^{t}\left|e^{-i \Gamma(s) \xi \cdot x}-1\right||h(s)|\left|\tilde{b}_{\mu}(x)\right| \frac{d s}{s} d \sigma(x)
$$

Hence, by (iii) and since $\Gamma$ is increasing we get

$$
\left|\widehat{\sigma}_{\widetilde{b}_{\mu}, t}(\xi)\right| \leq C|\Gamma(t) \xi|
$$

By using the same argument as above we get (2.10). The lemma is proved.

Lemma 2.8. Let $\mu \in \mathbf{N} \cup\{0\}, h \in \Delta_{\gamma}\left(\mathbf{R}_{+}\right)$for some $\gamma>1, \gamma^{\prime}<p<\infty$ and $\omega \in \widetilde{A}_{p / \gamma^{\prime}}\left(\mathbf{R}_{+}\right)$. Assume that $\tilde{b}_{\mu} \in L^{1}\left(\mathbf{S}^{n-1}\right)$ and $\Gamma$ satisfies either hypothesis $\mathbf{I}$ or hypothesis $\mathbf{D}$. Then there exists a positive constant $C_{p}$ such that

$$
\left\|\sigma_{\widetilde{b}_{\mu}}^{*}(f)\right\|_{L^{p}(\omega)} \leq C_{p}\left\|\tilde{b}_{\mu}\right\|_{L^{1}\left(s^{n-1}\right)}\|f\|_{L^{p}(\omega)}
$$

Proof. By Hölder's inequality, we have

$$
\begin{aligned}
& || \sigma_{\tilde{b}_{\mu}, t}|* f(x)| \\
& \leq\left(\int_{(1 / 2) t}^{t}|h(s)|^{\gamma} \frac{d s}{s}\right)^{1 / \gamma}\left(\int_{(1 / 2) t}^{t}\left|\int_{S^{n-1}} \tilde{b}_{\mu}\left(y^{\prime}\right) f\left(x-\Gamma(s) y^{\prime}\right) d \sigma\left(y^{\prime}\right)\right|^{\gamma^{\prime}} \frac{d s}{s}\right)^{1 / \gamma^{\prime}} \\
& \quad \leq C\left\|\tilde{b}_{\mu}\right\|_{L^{1}\left(\mathbf{S}^{n-1}\right)}^{1 / \gamma}\left(\int_{(1 / 2) t}^{t} \int_{S^{n-1}}\left|\tilde{b}_{\mu}\left(y^{\prime}\right)\right|\left|f\left(x-\Gamma(s) y^{\prime}\right)\right|^{\gamma^{\prime}} d \sigma\left(y^{\prime}\right) \frac{d s}{s}\right)^{1 / \gamma^{\prime}} .
\end{aligned}
$$

Thus

$$
\sigma_{\widetilde{b}_{\mu}}^{*} f(x) \leq C\left\|\widetilde{b}_{\mu}\right\|_{L^{1}\left(\mathbf{S}^{n-1}\right)}^{1 / \gamma}\left(\int_{S^{n-1}}\left|\tilde{b}_{\mu}\left(y^{\prime}\right)\right| M_{\Gamma, y^{\prime}}\left(|f|^{\gamma^{\prime}}\right)(x) d \sigma\left(y^{\prime}\right)\right)^{1 / \gamma^{\prime}}
$$

where

$$
M_{\Gamma, y^{\prime}} f(x)=\sup _{t \in \mathbf{R}_{+}}\left|\int_{t / 2}^{t} f\left(x-\Gamma(s) y^{\prime}\right) \frac{d s}{s}\right| .
$$


Let $w=\Gamma(s)$. Assume first that $\Gamma$ satisfies hypothesis $\mathbf{I}$. By the assumptions on $\Gamma$, we have $d s / s \leq d w / \alpha w$. So, by a change of variable we have

$$
\begin{aligned}
M_{\Gamma, y^{\prime}} f(x) & \leq \sup _{t \in \mathbf{R}_{+}}\left(\int_{\Gamma(t / 2)}^{\Gamma(t)}\left|f\left(x-w y^{\prime}\right)\right| \frac{d w}{w}\right) \\
& \leq \sup _{t \in \mathbf{R}_{+}}\left(\int_{\Gamma(t / 2)}^{c \Gamma(t / 2)}\left|f\left(x-w y^{\prime}\right)\right| \frac{d w}{w}\right) \\
& \leq C M_{y^{\prime}} f(x),
\end{aligned}
$$

where

$$
M_{y^{\prime}} f(x)=\sup _{R>0} R^{-1} \int_{0}^{R}\left|f\left(x-w y^{\prime}\right)\right| d w
$$

is the Hardy-Littlewood maximal function of $f$ in the direction of $y^{\prime}$. On the other hand, if $\Gamma$ satisfies hypothesis $\mathbf{D}$, as above we have $d s / s \leq-d w / \alpha w$ and

$$
\begin{aligned}
M_{\Gamma, y^{\prime}} f(x) & \leq \frac{1}{\alpha} \sup _{t \in \mathbf{R}_{+}}\left(\int_{\Gamma(t)}^{\Gamma(t / 2)}\left|f\left(x-w y^{\prime}\right)\right| \frac{d w}{w}\right) \\
& \leq \frac{1}{\alpha} \sup _{t \in \mathbf{R}_{+}}\left(\int_{(1 / c) \Gamma(t / 2)}^{\Gamma(t / 2)}\left|f\left(x-w y^{\prime}\right)\right| \frac{d w}{w}\right) \\
& \leq C M_{y^{\prime}} f(x) .
\end{aligned}
$$

By (2.26)-(2.30) and Minkowski's inequality for integrals we get

$$
\left\|\sigma_{\widetilde{b}_{\mu}}^{*}(f)\right\|_{L^{p}(\omega)} \leq C\left\|\tilde{b}_{\mu}\right\|_{L^{1}\left(S^{n-1}\right)}^{1 / \gamma}\left(\int_{S^{n-1}}\left|\tilde{b}_{\mu}\left(y^{\prime}\right)\right|\left\|M_{y^{\prime}}\left(|f|^{\gamma^{\prime}}\right)\right\|_{L^{p / \gamma^{\prime}}(\omega)} d \sigma\left(y^{\prime}\right)\right)^{1 / \gamma^{\prime}} .
$$

By $[6$, equation $(8)]$ and since $\omega \in \widetilde{A}_{p / \gamma^{\prime}}\left(\mathbf{R}_{+}\right)$we have

$$
\left\|M_{y^{\prime}} f\right\|_{L^{p / \gamma^{\prime}}(\omega)} \leq C\|f\|_{L^{p / \gamma^{\prime}}(\omega)}
$$

with $C$ independent of $y^{\prime}$. Thus, by (2.31)-(2.32) we get (2.24). This completes the proof of the lemma.

Lemma 2.9. Let $\mu \in \mathbf{N} \cup\{0\}, h \in \Delta_{\gamma}\left(\mathbf{R}_{+}\right)$for some $\gamma \geq 2, \gamma^{\prime}<p<\infty$ and $\omega \in \widetilde{A}_{p / \gamma^{\prime}}\left(\mathbf{R}_{+}\right)$. Assume that $\tilde{b}_{\mu} \in L^{1}\left(\mathbf{S}^{n-1}\right)$ and $\Gamma$ satisfies either hypothesis $\mathbf{I}$ or hypothesis $\mathbf{D}$. Then there exists a positive constant $C_{p}$ such that the inequality

$$
\begin{aligned}
& \left\|\left(\sum_{k \in \mathbf{Z}} \int_{\omega_{\mu}^{k}}^{\omega_{\mu}^{k+1}}\left|\sigma_{\tilde{b}_{\mu}, t} * g_{k}\right|^{2} \frac{d t}{t}\right)^{1 / 2}\right\|_{L^{p}(\omega)}\left\|\left(\sum_{k \in \mathbf{Z}}\left|g_{k}\right|^{2}\right)^{1 / 2}\right\|_{L^{p}(\omega)}, \\
& \quad \leq C_{p}\left(\log \left|I_{\mu}\right|^{-1}\right)^{1 / 2}\left\|\tilde{b}_{\mu}\right\|_{L^{1}\left(\mathbf{S}^{n-1}\right)}
\end{aligned}
$$

holds for any sequence of functions $\left\{g_{k}\right\}_{k \in \mathbf{Z}}$ on $\mathbf{R}^{n}$. 
Proof. Let $\gamma^{\prime}<p<\infty$. By a change of variable, we have

$$
\left(\sum_{k \in \mathbf{Z}} \int_{\omega_{\mu}^{k}}^{\omega_{\mu}^{k+1}}\left|\sigma_{\widetilde{b}_{\mu}, t} * g_{k}\right|^{2} \frac{d t}{t}\right)^{1 / 2} \leq\left(\sum_{k \in \mathbf{Z}} \int_{1}^{\omega_{\mu}}\left|\sigma_{\widetilde{b}_{\mu}, \omega_{\mu}^{k} t} * g_{k}\right|^{2} \frac{d t}{t}\right)^{1 / 2} .
$$

By Hölder's inequality and following a similar arguments as in the proof of (2.25) we get

$$
\left|\sigma_{\tilde{b}_{\mu}, t} * g_{k}(x)\right|^{\gamma^{\prime}} \leq C \mid\left\|\tilde{b}_{\mu}\right\|_{L^{1}\left(\mathbf{S}^{n-1}\right)}\left(\int_{t / 2}^{t} \int_{S^{n-1}}\left|\tilde{b}_{\mu}\left(y^{\prime}\right)\right|\left|g_{k}\left(x-\Gamma(s) y^{\prime}\right)\right|^{\gamma^{\prime}} d \sigma\left(y^{\prime}\right) \frac{d s}{s}\right) .
$$

Let $d=p / \gamma^{\prime}$. By duality, there is a nonnegative function $f \in L^{d^{\prime}}\left(\omega^{1-d^{\prime}}, \mathbf{R}^{n}\right)$ satisfying $\|f\|_{L^{d^{\prime}}\left(\omega^{1-d^{\prime}}\right)} \leq 1$ such that

$$
\left\|\left(\sum_{k \in \mathbf{Z}} \int_{1}^{\omega_{\mu}}\left|\sigma_{\widetilde{b}_{\mu}, \omega_{\mu}^{k} t} * g_{k}\right|^{\gamma^{\prime}} \frac{d t}{t}\right)^{1 / \gamma^{\prime}}\right\|_{L^{p}(\omega)}^{\gamma^{\prime}}=\int_{\mathbf{R}^{n}} \sum_{k \in \mathbf{Z}} \int_{1}^{\omega_{\mu}}\left|\sigma_{\widetilde{b}_{\mu}, \omega_{\mu}^{k} t} * g_{k}(x)\right|^{\gamma^{\prime}} \frac{d t}{t} f(x) d x .
$$

Therefore, by (2.35)-(2.36) and a change of variable we get

$$
\begin{aligned}
& \left\|\left(\sum_{k \in \mathbf{Z}} \int_{1}^{\omega_{\mu}}\left|\sigma_{\tilde{b}_{\mu}, \omega_{\mu}^{k} t} * g_{k}\right|^{\gamma^{\prime}} \frac{d t}{t}\right)^{1 / \gamma^{\prime}}\right\|_{L^{p}(\omega)}^{\gamma^{\prime}} \\
& \quad \leq C\left(\log \left|I_{\mu}\right|^{-1}\right)\left\|\tilde{b}_{\mu}\right\|_{L^{1}\left(\mathbf{S}^{n-1}\right)} \int_{\mathbf{R}^{n}} \sum_{k \in \mathbf{Z}}\left|g_{k}(x)\right|^{\gamma^{\prime}} M_{\mu}^{*} f(x) d x,
\end{aligned}
$$

where

$$
M_{\mu}^{*} f(x)=\sup _{t \in \mathbf{R}_{+}} \int_{(1 / 2) t<|y| \leq t} f\left(x+\Gamma(|y|) y^{\prime}\right)\left|\tilde{b}_{\mu}\left(y^{\prime}\right)\right||y|^{-n} d y .
$$

By Hölder's inequality, we obtain

$$
\begin{aligned}
& \left\|\left(\sum_{k \in \mathbf{Z}} \int_{1}^{\omega_{\mu}}\left|\sigma_{\tilde{b}_{\mu}, \omega_{\mu}^{k} t} * g_{k}\right|^{\gamma^{\prime}} \frac{d t}{t}\right)^{1 / \gamma^{\prime}}\right\|_{L^{p}(\omega)}^{\gamma^{\prime}} \\
& \quad \leq C\left(\log \left|I_{\mu}\right|^{-1}\right)\left\|\tilde{b}_{\mu}\right\|_{L^{1}\left(\mathbf{S}^{n-1}\right)}\left\|\left(\sum_{k \in \mathbf{Z}}\left|g_{k}\right|^{\gamma^{\prime}}\right)^{1 / \gamma^{\prime}}\right\|_{L^{p}(\omega)}^{\gamma^{\prime}}\left\|M_{\mu}^{*} f\right\|_{L^{d^{\prime}}\left(\omega^{1-d^{\prime}}\right)}
\end{aligned}
$$

It is easy to verify that $\omega \in \widetilde{A}_{d}\left(\mathbf{R}^{+}\right)$if and only if $\omega^{1-d^{\prime}} \in \widetilde{A}_{d^{\prime}}\left(\mathbf{R}^{+}\right)$. By the same argument as in the proof of Lemma 2.8, we have

$$
\left\|M_{\mu}^{*} f\right\|_{L^{d^{\prime}\left(\omega^{1-d^{\prime}}\right)}} \leq C_{p}\left\|\tilde{b}_{\mu}\right\|_{L^{1}\left(\mathbf{S}^{n-1}\right)}
$$


which in turn implies

$$
\begin{aligned}
& \left\|\left(\sum_{k \in \mathbf{Z}} \int_{1}^{\omega_{\mu}}\left|\sigma_{\tilde{b}_{\mu}, \omega_{\mu}^{k} t} * g_{k}\right|^{\gamma^{\prime}} \frac{d t}{t}\right)^{1 / \gamma^{\prime}}\right\|_{L^{p}(\omega)} \\
& \quad \leq C\left(\log \left|I_{\mu}\right|^{-1}\right)^{1 / \gamma^{\prime}}\left\|\tilde{b}_{\mu}\right\|_{L^{1}\left(\mathbf{S}^{n-1}\right)}^{2 / \gamma^{\prime}}\left\|\left(\sum_{k \in \mathbf{Z}}\left|g_{k}\right|^{\gamma^{\prime}}\right)^{1 / \gamma^{\prime}}\right\|_{L^{p}(\omega)} .
\end{aligned}
$$

On the other hand, by Lemma 2.8, we get

$$
\left\|\sup _{k \in \mathbf{Z}} \sup _{t \in\left[1, \omega_{\mu}\right]}\left|\sigma_{\widetilde{b}_{\mu}, \omega_{\mu}^{k} t} * g_{k}\right|\right\|_{L^{p}(\omega)} \leq\left\|\sigma^{*}\left(\sup _{k \in \mathbf{Z}}\left|g_{k}\right|\right)\right\|_{L^{p}(\omega)} \leq C\left\|\tilde{b}_{\mu}\right\|_{L^{1}\left(\mathbf{S}^{n-1}\right)}\left\|\sup _{k \in \mathbf{Z}}\left|g_{k}\right|\right\|_{L^{p}(\omega)} .
$$

Now, we define a linear operator $T$ on any function $g=g_{k}(x)$ by $T\left(g_{k}(x)\right)=\sigma_{\widetilde{b}_{\mu}, \omega_{\mu}^{k} t} *$ $g_{k}(x)$. Then by (2.41), we have

$$
\|\|\|T(g)\|_{L^{y^{\prime}}\left(\left[1, \omega_{\mu}\right], d t / t\right)}\left\|_{l^{\prime}(\mathbf{Z})}\right\|_{L^{p}\left(\mathbf{R}^{n}, \omega(x) d x\right)} \leq C\left(\log \left|I_{\mu}\right|^{-1}\right)^{1 / \gamma^{\prime}}\|\| g\left\|_{l^{y^{\prime}}(\mathbf{Z})}\right\|_{L^{p\left(\mathbf{R}^{n}, \omega(x) d x\right)}} .
$$

Also, by (2.42) we get

$$
\begin{aligned}
& \|\|\|\| T(g)\left\|\left.\right|_{L^{\infty}\left(\left[1, \omega_{\mu}\right], d t / t\right)}\right\|_{l^{\infty}(\mathbf{Z})} \|_{L^{p}\left(\mathbf{R}^{n}, \omega(x) d x\right)} \\
& \quad=\|\|\|T(g)\|\left\|_{L^{\infty}\left(\left[1, \omega_{\mu}\right], d t\right)}\right\|_{l^{\infty}(\mathbf{Z})} \|_{L^{p}\left(\mathbf{R}^{n}, \omega(x) d x\right)} \\
& \quad \leq C\|\| g\left\|_{l^{\infty}(\mathbf{Z})}\right\|_{L^{p}\left(\mathbf{R}^{n}, \omega(x) d x\right)} .
\end{aligned}
$$

Therefore, we can interpolate (2.43) and (2.44) (See [9, page 481] for the vector-valued interpolation) to get (2.33). The lemma is proved.

By following the same argument as in the proof of [1, Lemma 3.4], we get the following lemma.

Lemma 2.10. Let $\mu \in \mathbf{N} \cup\{0\}, h \in \Delta_{\gamma}\left(\mathbf{R}_{+}\right)$for some $\gamma \in(1,2]$. Assume that $\tilde{b}_{\mu} \in L^{1}\left(\mathbf{S}^{n-1}\right)$ and $\Gamma$ satisfies either hypothesis $\mathbf{I}$ or hypothesis $\mathbf{D}$. Then, for any $p$ satisfying $|1 / p-1 / 2|<$ $1 / \gamma^{\prime}$, there exists a positive constant $C_{p}$ such that

$$
\begin{aligned}
& \left\|\left(\sum_{k \in \mathbf{Z}} \int_{\omega_{\mu}^{k}}^{\omega_{\mu}^{k+1}}\left|\sigma_{\widetilde{b}_{\mu}, t} * g_{k}\right|^{2} \frac{d t}{t}\right)^{1 / 2}\right\|_{L^{p}\left(\mathbf{R}^{n}\right)} \\
& \quad \leq C_{p}\left(\log \left|I_{\mu}\right|^{-1}\right)^{1 / 2}\left\|\tilde{b}_{\mu}\right\|_{L^{1}\left(\mathbf{S}^{n-1}\right)}\left\|\left(\sum_{k \in \mathbf{Z}}\left|g_{k}\right|^{2}\right)^{1 / 2}\right\|_{L^{p}\left(\mathbf{R}^{n}\right)}
\end{aligned}
$$

holds for arbitrary functions $\left\{g_{k}(\cdot)\right\}_{k \in \mathbf{Z}}$ on $\mathbf{R}^{n}$. The constant $C_{p}$ is independent of $\mu$. 


\section{Proofs of main results}

We will only present the proof of Theorems 1.3 and 1.4 for the case $\Gamma$ satisfies hypothesis $\mathbf{I}$, since the proofs for the case $\Gamma$ satisfies hypothesis $\mathbf{D}$ are essentially the same. Assume that $\Omega \in B_{q}^{(0,-1 / 2)}\left(\mathbf{S}^{n-1}\right)$ for some $q>1$ and satisfies (1.1). Thus $\Omega$ can be written as $\Omega=\sum_{\mu=1}^{\infty} \lambda_{\mu} b_{\mu}$, where $\lambda_{\mu} \in \mathbf{C}, b_{\mu}$ is a $q$-block supported on a cap $I_{\mu}$ on $\mathbf{S}^{n-1}$ and $M_{q}^{(0,-1 / 2)}\left(\left\{\lambda_{\mu}\right\}\right)<\infty$. To each block function $b_{\mu}(\cdot)$, let $\tilde{b}_{\mu}(\cdot)$ be a function defined by

$$
\tilde{b}_{\mu}(x)=b_{\mu}(x)-\int_{S^{n-1}} b_{\mu}(u) d \sigma(u)
$$

Let $\mathbf{J}=\left\{\mu \in \mathbf{N}:\left|I_{\mu}\right|<e^{-2}\right\}$. Let $\widetilde{b}_{0}=\Omega-\sum_{\mu \in \mathbf{J}}^{\infty} \eta_{\mu} \widetilde{b}_{\mu}$. Then for some positive constant $C$, the following holds for all $\mu \in \mathbf{J} \cup\{0\}$ :

$$
\begin{gathered}
\int_{\mathbf{S}^{n-1}} \tilde{b}_{\mu}(u) d \sigma(u)=0, \\
\left\|\tilde{b}_{\mu}\right\|_{q} \leq C\left|I_{\mu}\right|^{-1 / q^{\prime}}, \\
\left\|\tilde{b}_{\mu}\right\|_{1} \leq C, \\
\Omega=\sum_{\mu \in \mathrm{J} \cup\{0\}} \lambda_{\mu} \tilde{b}_{\mu},
\end{gathered}
$$

where $I_{0}$ is a cap on $\mathrm{S}^{n-1}$ with $\left|I_{0}\right|=e^{-3}$.

By (3.5) we have

$$
M_{\Omega, \Gamma, h}^{\rho}(f) \leq \sum_{\mu \in \mathrm{J} \cup\{0\}}\left|\lambda_{\mu}\right| M_{\tilde{b}_{\mu}, \Gamma, h}^{\rho}(f) .
$$

Therefore, Theorems 1.3 and 1.4 are proved if we can show that

$$
\left\|\mathcal{M}_{\tilde{b}_{\mu}, \Gamma, h}^{\rho}(f)\right\|_{L^{p}\left(\mathbf{R}^{n}\right)} \leq C_{p}\left(\log \left|I_{\mu}\right|^{-1}\right)^{1 / 2}\|f\|_{L^{p}\left(\mathbf{R}^{n}\right)}
$$

for $p$ satisfying $|1 / p-1 / 2|<\min \left\{1 / \gamma^{\prime}, 1 / 2\right\}$; and

$$
\left\|\mu_{\widetilde{b}_{\mu}, \Gamma, h}^{\rho}(f)\right\|_{L^{p}(\omega)} \leq C_{p}\left(\log \left|I_{\mu}\right|^{-1}\right)^{1 / 2}\|f\|_{L^{p}(\omega)}
$$

for all $\omega \in \widetilde{A}_{p / \gamma^{\prime}}^{I}\left(\mathbf{R}_{+}\right)$and $\gamma^{\prime}<p<\infty$.

Proof of (3.7). Since $\Delta_{\gamma}\left(\mathbf{R}_{+}\right) \subseteq \Delta_{2}\left(\mathbf{R}_{+}\right)$for $\gamma \geq 2$, we may assume that $1<\gamma \leq 2$. Therefore, it suffices to prove (3.7) for $p$ satisfying $|1 / p-1 / 2|<1 / \gamma^{\prime}$. Let $\left\{\phi_{k, \mu}\right\}_{-\infty}^{\infty}$ be a smooth partition of unity in $(0, \infty)$ adapted to the interval $\mathscr{E}_{k, \mu}=\left[a_{k+1, \mu}^{-1}, a_{k-1, \mu}^{-1}\right]$. To be precise, we 
require the following:

$$
\begin{gathered}
\phi_{k, \mu} \in C^{\infty}, 0 \leq \phi_{k, \mu} \leq 1, \quad \sum_{k} \phi_{k, \mu}(t)=1 ; \\
\operatorname{supp} \phi_{k, \mu} \subseteq \mathscr{F}_{k, \mu} ; \quad\left|\frac{d^{s} \phi_{k, \mu}(t)}{d t^{s}}\right| \leq \frac{C_{s}}{t^{s}}
\end{gathered}
$$

where $C_{s}$ is independent of the lacunary sequence $\left\{a_{\mu, k}: k \in \mathbf{Z}\right\}$. Let $\widehat{\Upsilon_{k, \mu}}(\xi)=\phi_{k, \mu}(|\xi|)$.

By Minkowski's inequality we have

$$
\begin{aligned}
\mu_{\tilde{b}_{\mu}, \Gamma, h}^{\rho} f(x) & =\left(\int_{0}^{\infty}\left|\sum_{k=0}^{\infty} 2^{-k \rho} \sigma_{\widetilde{b}_{\mu}, 2^{-k} t} * f(x)\right|^{2} \frac{d t}{t}\right)^{1 / 2} \\
& \leq \sum_{k=0}^{\infty} 2^{-k \sigma}\left(\int_{0}^{\infty}\left|\sigma_{\widetilde{b}_{\mu}, 2-k t} * f(x)\right|^{2} \frac{d t}{t}\right)^{1 / 2} \\
& =C_{\sigma}\left(\int_{0}^{\infty}\left|\sigma_{\widetilde{b}_{\mu}, t} * f(x)\right|^{2} \frac{d t}{t}\right)^{1 / 2} .
\end{aligned}
$$

Decompose

$$
f * \sigma_{\widetilde{b}_{\mu}, t}(x)=\sum_{j \in \mathbf{Z}} \sum_{k \in \mathbf{Z}}\left(\Upsilon_{k+j, \mu} * \sigma_{\widetilde{b}_{\mu}, t} * f\right)(x) \chi_{\left[\omega_{\mu}^{k}, \omega_{\mu}^{k+1}\right)}(t):=\sum_{j \in \mathbf{Z}} G_{j, \mu}(x, t)
$$

and define

$$
T_{j, \mu} f(x)=\left(\int_{0}^{\infty}\left|G_{j, \mu}(x, t)\right|^{2} \frac{d t}{t}\right)^{1 / 2}
$$

Then

$$
\mathcal{M}_{\tilde{b}_{\mu}, \Gamma, h}^{\rho}(f) \leq C_{\sigma} \sum_{j \in \mathbf{Z}} T_{j, \mu}(f)
$$

holds for $f \in \mathscr{S}\left(\mathbf{R}^{n}\right)$. Thus, to prove (3.7), it is enough to show that

$$
\left\|T_{j, \mu}(f)\right\|_{L^{p}\left(\mathbf{R}^{n}\right)} \leq C_{p}\left(\log \left|I_{\mu}\right|^{-1}\right)^{1 / 2} \eta^{-\alpha_{p}|j|}\|f\|_{L^{p}\left(\mathbf{R}^{n}\right)}
$$

for some $\alpha_{p}>0$ and for $p$ satisfying $|1 / p-1 / 2|<1 / \gamma^{\prime}$.

To prove (3.14), let us first compute the $L^{2}$-norm of $T_{j, \mu}(f)$. By using Plancherel's theorem, we have

$$
\begin{aligned}
\left\|T_{j, \mu}(f)\right\|_{L^{2}\left(\mathbf{R}^{n}\right)}^{2} & =\sum_{k \in \mathbf{Z}} \int_{\mathbf{R}^{n}} \int_{\omega_{\mu}^{k}}^{\omega_{\mu}^{k+1}}\left|\Upsilon_{k+j, \mu} * \sigma_{\widetilde{b}_{\mu}, t} * f(x)\right|^{2} \frac{d t}{t} d x \\
& \leq \sum_{k \in \mathbf{Z}} \int_{\eta_{k+j, \mu}}\left(\int_{\omega_{\mu}^{k}}^{\omega_{\mu}^{k+1}}\left|\hat{\sigma}_{\widetilde{b}_{\mu}, t}(\xi)\right|^{2} \frac{d t}{t}\right)|\hat{f}(\xi)|^{2} d \xi,
\end{aligned}
$$

where

$$
\eta_{k, \mu}=\left\{\xi \in \mathbf{R}^{n}:|\xi| \in \mathscr{J}_{k, \mu}\right\}
$$


By Lemma 2.7 we have

$$
\left\|T_{j, \mu}(f)\right\|_{L^{2}\left(\mathbf{R}^{n}\right)} \leq C\left(\log \left|I_{\mu}\right|^{-1}\right)^{1 / 2} \eta^{-\beta|j|}\|f\|_{L^{2}\left(\mathbf{R}^{n}\right)} .
$$

Next, let us compute the $L^{p}$ boundedness of the operator $T_{j, \mu}$. For $|1 / p-1 / 2|<1 / \gamma^{\prime}$, we have

$$
\begin{aligned}
\left\|T_{j, \mu}(f)\right\|_{L^{p}\left(\mathbf{R}^{n}\right)} & \leq C_{p}\left(\log \left|I_{\mu}\right|^{-1}\right)^{1 / 2}\left\|\left(\sum_{k \in \mathbf{Z}}\left|\Upsilon_{k+j, \mu} * f\right|^{2}\right)^{1 / 2}\right\|_{L^{p}\left(\mathbf{R}^{n}\right)} \\
& \leq C_{p}\left(\log \left|I_{\mu}\right|^{-1}\right)^{1 / 2}\|f\|_{L^{p}\left(\mathbf{R}^{n}\right) .}
\end{aligned}
$$

The last two inequalities are obtained by applying Lemma 2.10 and applying the Littlewood-Paley theory and Theorem 3 along with the remark that follows its statement in [18, page 96$]$.

Now by interpolation between (3.17) and (3.18) we get (3.14). This completes the proof of Theorem 1.3.

Proof of (3.8). Assume that $\omega \in \widetilde{A}_{p / \gamma^{\prime}}^{I}\left(\mathbf{R}_{+}\right)$and $p>\gamma^{\prime}$. As above, to prove (3.8), it suffices to show that

$$
\left\|T_{j, \mu}(f)\right\|_{L^{p}(\omega)} \leq C\left(\log \left|I_{\mu}\right|^{-1}\right)^{1 / 2} \eta^{-\alpha_{p}|j|}\|f\|_{L^{p}(\omega)}
$$

To this end, let us compute the $L^{p}(\omega)$ norm of $T_{j, \mu}(f)$. For all $\omega \in \widetilde{A}_{p / \gamma^{\prime}}^{I}\left(\mathbf{R}_{+}\right)$and $\gamma^{\prime}<p<$ $\infty$, we have

$$
\begin{aligned}
\left\|T_{j, \mu}(f)\right\|_{L^{p}(\omega)} & \leq C_{p}\left(\log \left|I_{\mu}\right|^{-1}\right)^{1 / 2}\left\|\left(\sum_{k \in \mathbf{Z}}\left|\Upsilon_{k+j, \mu} * f\right|^{2}\right)^{1 / 2}\right\|_{L^{p}(\omega)} \\
& \leq C_{p}\left(\log \left|I_{\mu}\right|^{-1}\right)^{1 / 2}\|f\|_{L^{p}(\omega)},
\end{aligned}
$$

where the first inequality follows by Lemma 2.9 and the last inequality follows from a well-known weighted Littlewood-Paley inequality since $\omega \in \tilde{A}_{p / \gamma^{\prime}}\left(\mathbf{R}_{+}\right) \subset A_{p}\left(\mathbf{R}_{+}\right)$(see [12]). By interpolating between (3.17) and (3.20) with $\omega=1$ we get

$$
\left\|T_{j, \mu}(f)\right\|_{L^{p}\left(\mathbf{R}^{n}\right)} \leq C_{p}\left(\log \left|I_{\mu}\right|^{-1}\right)^{1 / 2} \eta^{-\alpha_{p}|j|}\|f\|_{L^{p}\left(\mathbf{R}^{n}\right)} \quad \text { for } \gamma^{\prime}<p<\infty .
$$

By Lemma 2.3, for any $\omega \in \tilde{A}_{p / \gamma^{\prime}}^{I}\left(\mathbf{R}_{+}\right)$, there is an $\varepsilon>0$ such that $\omega^{1+\varepsilon} \in \tilde{A}_{p / \gamma^{\prime}}^{I}\left(\mathbf{R}_{+}\right)$. Thus

$$
\left\|T_{j, \mu}(f)\right\|_{L^{p}\left(\omega^{1+\varepsilon}\right)} \leq C_{p}\left(\log \left|I_{\mu}\right|^{-1}\right)^{1 / 2}\|f\|_{L^{p}\left(\omega^{1+\varepsilon}\right)} \quad \text { for } \gamma^{\prime}<p<\infty
$$

By interpolating with change of measures between (3.20) and (3.21) we get (3.19).

\section{Further results}

As an application of Theorem 1.4, we get the weighted $L^{p}$ boundedness for a class of parametric Marcinkiewicz operators $\mathcal{M}_{\Omega, \Gamma, h, \lambda}^{*, \rho}$ and $\mathcal{M}_{\Omega, \Gamma, h, S}^{\rho}$ related to the Littlewood-Paley 
$g_{\eta}^{*}$-function and the area integral $S$, respectively. The definition and the precise statement of the results regarding these operators are given as follows.

Theorem 4.1. Let $h \in \Delta_{\gamma}\left(\mathbf{R}_{+}\right)$with $\gamma \geq 2,1<p<\infty$. Assume $\Gamma$ satisfies either hypothesis I or hypothesis $\mathbf{D}$ and $\Omega \in B_{q}^{(0,-1 / 2)}\left(\mathbf{S}^{n-1}\right)$. Then there exists $C_{p}>0$ such that

$$
\left\|M_{\Omega, \Gamma, h, S}^{\rho}(f)\right\|_{L^{p}(\omega)}+\left\|M_{\Omega, \Gamma, h, \lambda}^{\rho, *}(f)\right\|_{L^{p}(\omega)} \leq C_{p}\|\Omega\|_{B_{q}^{(0,-1 / 2)}\left(S^{n-1}\right)}\|f\|_{L^{p}(\omega)}
$$

for $2 \leq p<\infty$ and $\omega \in \tilde{A}_{p / 2}^{I}\left(\mathbf{R}_{+}\right)$. Here $\mathcal{M}_{\Omega, \Gamma, h, S}^{\rho}$ are $M_{\Omega, \Gamma, h, \lambda}^{*, \rho}$ are defined by

$$
\begin{gathered}
\mathcal{M}_{\Omega, \Gamma, h, S}^{\rho} f(x)=\left(\int_{\Gamma(x)}\left|F_{\Omega, \Gamma, h}^{\rho} f(t, y)\right|^{2} \frac{d y d t}{t^{n+1}}\right)^{1 / 2} \\
\mathcal{M}_{\Omega, \Gamma, h, \lambda}^{*, \rho} f(x)=\left(\int_{\mathbf{R}_{+}^{n+1}}\left(\frac{t}{t+|x-y|}\right)^{n \eta}\left|F_{\Omega, \Gamma, h}^{\rho} f(t, y)\right|^{2} \frac{d y d t}{t^{n+1}}\right)^{1 / 2},
\end{gathered}
$$

where $\eta>1$ and $\Gamma(x)=\left\{(y, t) \in \mathbf{R}_{+}^{n+1}:|x-y|<t\right\}$.

The proof of Theorem 4.1 is based on the following lemma.

Lemma 4.2. Let $\lambda>1$. Then, for any nonnegative locally integrable function $g$, we have

$$
\int_{\mathbf{R}^{n}}\left(M_{\Omega, \Psi, h, \lambda}^{\rho, *} f(x)\right)^{2} g(x) d x \leq C_{\sigma} \int_{\mathbf{R}^{n}}|f(x)|^{2} M g(x) d x .
$$

A proof of this lemma can be obtained by Theorem 1.3 and following a similar argument as in the proof of Theorem 5 in Torchinsky and Wang [19].

Proof of Theorem 4.1. Since $M_{\Omega, \Psi, h, S}^{\rho} f(x) \leq 2^{n \lambda / 2} M_{\Omega, \Psi, h, \lambda}^{\rho, *} f(x)$, we only consider the operator $M_{\Omega, \Psi, h, \lambda}^{\rho, *}$. If $p=2$, we have $\omega \in \widetilde{A}_{1}^{I}\left(\mathbf{R}_{+}\right) \subset \widetilde{A}_{1}\left(\mathbf{R}_{+}\right) \subset A_{1}\left(\mathbf{R}_{+}\right)$and hence $M \omega(x) \leq$ $C \omega(x)$ a.e. Therefore, by Lemma 4.2 we have

$$
\int_{\mathbf{R}^{n}}\left(M_{\Omega, \Psi, h, \lambda}^{\rho, *} f(x)\right)^{2} \omega(x) d x \leq C \int_{\mathbf{R}^{n}}|f(x)|^{2} \omega(x) d x,
$$

and hence we get $\mathcal{M}_{\Omega, \Psi, h, \lambda}^{\rho, *}$ is bounded on $L^{2}(\omega)$. When $2<p<\infty$, we set $d=p / 2$. By Lemma 4.2 and Hölder's inequality we get

$$
\begin{aligned}
\left\|M_{\Omega, \Psi, h, \lambda}^{\rho, *} f\right\|_{L^{p}(\omega)}^{2} & =\sup _{g}\left|\int_{\mathbf{R}^{n}}\left(M_{\Omega, \Psi, h, \lambda}^{\rho, *} f(x)\right)^{2} g(x) d x\right| \\
& \leq C_{\sigma} \sup _{g} \int_{\mathbf{R}^{n}}|f(x)|^{2} M(|g|)(x) d x \\
& \leq C_{\sigma}\|f\|_{L^{p}(\omega)}^{2} \sup _{g}\|M g\|_{L^{d^{\prime}}\left(\omega^{1-d^{\prime}}\right)} \\
& \leq C_{\sigma}\|f\|_{L^{p}(\omega)}^{2},
\end{aligned}
$$

where the supremum is taken over all functions $g$ satisfying $\|g\|_{L^{d^{\prime}}\left(\omega^{1-d^{\prime}}\right)} \leq 1$. Hence, the proof of Theorem 4.1 is completed. 
One of the important special classes of radial weights is the power weights $|x|^{\alpha}, \alpha \in \mathbf{R}$. It is know that $|x|^{\alpha} \in A_{p}\left(\mathbf{R}^{n}\right)$ if and only if $-n<\alpha<n(p-1)$.

Our result regarding this class of weights is the following theorem.

Theorem 4.3. Let $h \in \Delta_{y}\left(\mathbf{R}^{+}\right)$with $\gamma \geq 2$. Assume $\Gamma$ satisfy either hypothesis $\mathbf{I}$ or hypothesis D. If $\Omega \in B_{q}^{(0,-1 / 2)}\left(\mathbf{S}^{n-1}\right)$, and $\gamma^{\prime}<p<\infty$, then

$$
\left\|\mathcal{M}_{\Omega, \Gamma, h}^{\rho}(f)\right\|_{L^{p}(\omega)} \leq\|f\|_{L^{p}(\omega)}
$$

for all $\omega(x)=|x|^{\alpha}$ and $\alpha \in\left(-1, p / \gamma^{\prime}-1\right)$. Similar results hold regarding the operators $M_{\Omega, \Psi, h, S}^{\rho}$ and $M_{\Omega, \Psi, h, \lambda}^{\rho, *}$.

A proof of this theorem can be obtained by Theorem 4.3 and noticing that $|x|^{\alpha} \in$ $\tilde{A}_{p}^{I}\left(\mathbf{R}_{+}\right)$for $\alpha \in(-1, p-1)$.

\section{Acknowledgment}

I would like to thank Professor Chin-Cheng Lin for sending me a preprint of his paper [13] and for his encouraging comments.

\section{References}

[1] H. M. Al-Qassem, $L^{p}$ estimates for rough parametric Marcinkiewicz integrals, SUT Journal of Mathematics 40 (2004), no. 2, 117-131.

[2] H. M. Al-Qassem and A. J. Al-Salman, A note on Marcinkiewicz integral operators, Journal of Mathematical Analysis and Applications 282 (2003), no. 2, 698-710.

[3] A. Benedek, A.-P. Calderón, and R. Panzone, Convolution operators on Banach space valued functions, Proceedings of the National Academy of Sciences of the United States of America 48 (1962), 356-365.

[4] J. Chen, D. Fan, and Y. Pan, A note on a Marcinkiewicz integral operator, Mathematische Nachrichten 227 (2001), no. 1, 33-42.

[5] Y. Ding, D. Fan, and Y. Pan, Weighted boundedness for a class of rough Marcinkiewicz integrals, Indiana University Mathematics Journal 48 (1999), no. 3, 1037-1055.

[6] J. Duoandikoetxea, Weighted norm inequalities for homogeneous singular integrals, Transactions of the American Mathematical Society 336 (1993), no. 2, 869-880.

[7] J. Duoandikoetxea and J. L. Rubio de Francia, Maximal and singular integral operators via Fourier transform estimates, Inventiones Mathematicae 84 (1986), no. 3, 541-561.

[8] D. Fan, Y. Pan, and D. Yang, A weighted norm inequality for rough singular integrals, The Tohoku Mathematical Journal. Second Series 51 (1999), no. 2, 141-161.

[9] J. García-Cuerva and J. L. Rubio de Francia, Weighted Norm Inequalities and Related Topics, North-Holland Mathematics Studies, vol. 116, North-Holland, Amsterdam, 1985.

[10] L. Hörmander, Estimates for translation invariant operators in $L^{p}$ spaces, Acta Mathematica 104 (1960), 93-140.

[11] M. Keitoku and E. Sato, Block spaces on the unit sphere in $\mathbf{R}^{n}$, Proceedings of the American Mathematical Society 119 (1993), no. 2, 453-455.

[12] D. S. Kurtz, Littlewood-Paley and multiplier theorems on weighted $L^{p}$ spaces, Transactions of the American Mathematical Society 259 (1980), no. 1, 235-254.

[13] M.-Y. Lee and C.-C. Lin, Weighted L $L^{p}$ boundedness of Marcinkiewicz integral, Integral Equations and Operator Theory 49 (2004), no. 2, 211-220. 
[14] S. Lu, M. Taibleson, and G. Weiss, Spaces Generated by Blocks, Normal University Press, Beijing, 1989.

[15] M. Sakamoto and K. Yabuta, Boundedness of Marcinkiewicz functions, Studia Mathematica 135 (1999), no. 2, 103-142.

[16] S. Sato, Remarks on square functions in the Littlewood-Paley theory, Bulletin of the Australian Mathematical Society 58 (1998), no. 2, 199-211.

[17] E. M. Stein, On the functions of Littlewood-Paley, Lusin, and Marcinkiewicz, Transactions of the American Mathematical Society 88 (1958), 430-466.

[18] Singular Integrals and Differentiability Properties of Functions, Princeton Mathematical Series, no. 30, Princeton University Press, New Jersey, 1970.

[19] A. Torchinsky and S. L. Wang, A note on the Marcinkiewicz integral, Colloquium Mathematicum 60/61 (1990), no. 1, 235-243.

H. M. Al-Qassem: Department of Mathematics, Yarmouk University, Irbid-Jordan, Jordan

E-mail address: husseink@yu.edu.jo 